\title{
p53 induces ARTS to promote mitochondrial apoptosis
}

Qian Hao ${ }^{1,2}$, Jiaxiang Chen ${ }^{3,8}$, Junming Liao ${ }^{3}$, Yingdan Huang ${ }^{1,2}$, Yu Gan ${ }^{4}$, Sarit Larisch $\mathbb{0}^{5}$, Shelya X. Zeng ${ }^{3}$, Hua Lu ${ }^{3}$ and Xiang Zhou (1D) $1,2,6,7$

\begin{abstract}
Apoptosis related protein in TGF- $\beta$ signaling pathway (ARTS) was originally discovered in cells undergoing apoptosis in response to TGF- $\beta$, but ART $\bar{S}$ also acts downstream of many other apoptotic stimuli. ARTS induces apoptosis by antagonizing the anti-apoptotic proteins XIAP and BCl-2. Here we identified the pro-apoptotic Sept4/ARTS gene as a p53-responsive target gene. Ectopic p53 and a variety of p53-inducing agents increased both mRNA and protein levels of ARTS, whereas ablation of p53 reduced ARTS expression in response to multiple stress conditions. Also, $y$-irradiation induced p53-dependent ARTS expression in mice. Consistently, p53 binds to the responsive DNA element on the ARTS promoter and transcriptionally activated the promoter-driven expression of a luciferase reporter gene. Interestingly, ARTS binds to and sequesters p53 at mitochondria, enhancing the interaction of the latter with BCl-XL. Ectopic ARTS markedly augments DNA damage stress- or Nutlin-3-triggered apoptosis, while ablation of ARTS preferentially impairs p53-induced apoptosis. Altogether, these findings demonstrate that ARTS collaborates with p53 in mitochondriaengaged apoptosis.
\end{abstract}

\section{Introduction}

The tumor-suppressor p53 prevents genomic instability and tumorigenesis through multiple mechanisms. As a transcription factor, it arrests cell cycle progression and facilitates DNA repair by inducing the RNA expression of a number of cell cycle- and DNA repair-associated genes when pre-cancerous cells undergo mild insult of replication stress. In addition, p53 can elicit cell death through upregulation of the pro-apoptotic genes, such as BAX, NOXA, and PUMA, when cancer cells are subjected to severe DNA damage stress, such as therapeutic intervention $^{1,2}$. However, independently of its transcriptional activity, p53 has also been shown to promote apoptosis via

\footnotetext{
Correspondence: Hua Lu (hlu2@tulane.edu) or Xiang Zhou (xiangzhou@fudan. edu.cn)

${ }^{1}$ Fudan University Shanghai Cancer Center and Institutes of Biomedical Sciences, Fudan University, Shanghai 200032, China

${ }^{2}$ Department of Oncology, Shanghai Medical College, Fudan University, Shanghai 200032, China

Full list of author information is available at the end of the article

These authors contributed equally: Qian Hao, Jiaxiang Chen

Edited by I. Amelio
}

mitochondria-involved mechanisms ${ }^{3}$. p53 was shown to bind to Bcl-XL via its DNA-binding domain and derepresses the mitochondrial BAK, BAX, and PUMA, consequently leading to mitochondrial outer membrane permeabilization and release of cytochrome $\mathrm{c}^{4-6} \cdot \mathrm{p} 53$ is such detrimental to cancer cells that several autoregulatory mechanisms have been evolved in cancer cells to control its activity ${ }^{7}$. The E3-ubiquitin ligase MDM2, encoded by a p53 target gene, is the master negative regulator that can inhibit p53 activity by directly concealing its transcriptional activation domain and promoting its proteolytic degradation ${ }^{8-11}$. Other p53inducible proteins, such as NGFR and PHLDB3 (refs. ${ }^{12,13}$ ), have been shown to either directly or collaborate with MDM2 to repress p53 as negative feedback regulators. This study as presented here identified ARTS (apoptosis-related protein in the TGF- $\beta$ signaling pathway) as another p53 target that could play a role in regulation of p53's apoptotic activity as well.

ARTS is a pro-apoptotic protein located at the outer membrane of the mitochondria ${ }^{14,15}$. ARTS protein is

\section{(c) The Author(s) 2021}

(c) (i) Open Access This article is licensed under a Creative Commons Attribution 4.0 International License, which permits use, sharing, adaptation, distribution and reproduction in any medium or format, as long as you give appropriate credit to the original author(s) and the source, provide a link to the Creative Commons license, and indicate if changes were made. The images or other third party material in this article are included in the article's Creative Commons license, unless indicated otherwise in a credit line to the material. If material is not included in the article's Creative Commons license and your intended use is not permitted by statutory regulation or exceeds the permitted use, you will need to obtain permission directly from the copyright holder. To view a copy of this license, visit http://creativecommons.org/licenses/by/4.0/. 
derived from alternative splicing of the SEPT4 gene and is the only isoform that can evoke mitochondrial apoptosis $^{14,16}$. Although ARTS was originally discovered in cells induced for apoptosis by transforming growth factor (TGF)- $\beta$, it was later found that ARTS acts downstream of basically all apoptosis stimuli tested, such as treatment with STS (staurosporine), etoposide, arabinoside (Ara-c), nocadosole, UV radiation, tumor necrosis factor- $\alpha$, etc. $^{14,17,18}$. ARTS initiates caspase activation upstream of mitochondria by directly binding and degrading XIAP (Xlinked inhibitor of apoptosis) via the ubiquitin proteasome system (UPS) ${ }^{15,19,20}$. Recently, ARTS was shown to induce ubiquitination and degradation of $\mathrm{Bcl}-2$ by bridging the E3-ubiquitin ligase XIAP to $\mathrm{Bcl}-2$ (ref. ${ }^{21}$ ). Studies in human and mice have shown that ARTS functions as a tumor-suppressor protein ${ }^{17,22-25}$. Moreover, Sept4/ARTSdeficient mice exhibit high levels of stem and progenitor cells, which are resistant to apoptosis ${ }^{23-25}$.

When screening for novel p53 target genes by microarray analysis of Inauhzin (INZ)-treated cancer cells ${ }^{26}$ [of note, INZ is a p53 activating small molecule identified by our laboratory ${ }^{27}$ ], we identified ARTS as a potential p53 target gene. Our further study of this molecule not only confirmed that p53 transcriptionally induces ARTS expression in cancer cells and in mice but also revealed that ARTS cooperates with p53 in inhibition of Bcl-XL in the mitochondria, consequently augmenting p53-dependent apoptosis. As detailed below, our findings demonstrate that p53-inducible ARTS can enhance p53-directed mitochondrial apoptosis.

\section{Materials and methods}

\section{Plasmids and antibodies}

The Flag-tagged pcDNA-ARTS plasmid was generated by inserting the full-length ARTS cDNA amplified from the pcDNA3-Myc-ARTS plasmid as a gift from Dr. Sarit Larisch into the pcDNA3.0/Flag vector. The plasmid encoding Flag-Bcl-XL was purchased from OriGene Technologies (Rockville, MD, USA). The plasmids encoding p53, HA-MDM2, and His-Ub were described previously $^{28}$. The pGL3-RE1, RE2, and RE3 plasmids were generated by inserting the genomic DNA covering p53 RE1, RE2 or RE3 into the pGL3-promoter vector using the following primers, 5'-CGGGGTACCATTCAGCAGGTG CCAGGAA-3' and 5'-CCGCTCGAGACGATACAGTCA GAGAGTCCTT-3' for RE1; 5'-CGGGGTACCGTATTA GACCCTGCCTCCATCA-3' and 5'-CCGCTCGAGGAA GACTGACTTTGAGCCATCC-3' for RE2; 5'-CGGGG TACCTGCCTCGGACTCCTGAGTA-3' and 5'-CCGCT CGAGGGGACAGACAAGCAGAGAAAC-3' for RE3. The lentivirus-based ARTS-overexpressing or short hairpin RNA (shRNA) plasmid was constructed using the vectors pLenti-EF1a-EGFP-P2A-Puro-CMV-3Flag and pLKD-CMV-G\&PR-U6, respectively (OBio Technology, Shanghai, China). The shRNA sequence targeting ARTS was previously described ${ }^{15}$. The anti-ARTS (Cat. No. A4471, Sigma-Aldrich, St Louis, MO, USA), anti-p53 (Cat. No. sc-126, Santa Cruz Biotechnology, Santa Cruz, CA, USA), anti-Flag (Cat. No. F1804, Sigma-Aldrich), anti-HA (Cat. No. 2367, Cell Signaling Technology, Danvers, MA, USA), anti-p21 (Cat. No. 2947, Cell Signaling Technology), anti-MDM2 (Cat. No. ab16895, 2A10, Abcam, Cambridge, MA, USA), anti-Bcl-XL (Cat. No. 10783-1-AP, Proteintech, Wuhan, Hubei, China), anti-cleaved poly ADP-ribose polymerase (PARP; Cat. No. 5625, Cell Signaling Technology), anti-COX IV (Cat. No. 11242-1-AP, Proteintech), anti-GAPDH (Cat. No. 600041-Ig, Proteintech), anti- $\beta$-actin (Cat. No. ARG62346, Proteintech), anti- $\alpha$-Tubulin (Cat. No. 66031-1-Ig, Proteintech), anti-Vinculin (Cat. No. 66306-1-Ig, Proteintech), anti-Lamin B (Cat. No. 12255, Cell Signaling Technology), and anti-histone H3 (Cat. No. 17168-1-AP, Proteintech) were commercially purchased.

\section{Cell culture and transient transfection}

Human cancer cell lines H460 and H1299 were purchased from American Type Culture Collection. HCT $116^{\mathrm{p}^{53+/+}}$ and HCT116 $6^{\mathrm{p} 53-/-}$ were generous gifts from Dr. Bert Vogelstein at the Johns Hopkins Medical Institute. SK-MEL-147 was a generous gift from Dr. Shaomeng Wang at University of Michigan, Ann Arbor. Cells were cultured in Dulbecco's modified Eagle's medium supplemented with $10 \%$ fetal bovine serum, $50 \mathrm{U} / \mathrm{ml}$ penicillin, and $0.1 \mathrm{mg} / \mathrm{ml}$ streptomycin and maintained at $37^{\circ} \mathrm{C}$ in a $5 \% \mathrm{CO}_{2}$ humidified atmosphere. All the cell lines were mycoplasma free and authenticated by PCR analysis. Cells seeded on the plate overnight were transfected with plasmids or small interfering RNA (siRNA) as indicated in the figure legends using Hieff TransTM Liposomal transfection reagent following the manufacturer's protocol (Yeasen, Shanghai, China). Cells were harvested at 30-72 h post-transfection for designed experiments. The proteasome inhibitor MG132 was purchased from Sigma-Aldrich.

\section{RNA interference}

siRNA against p53 was commercially purchased (GenePharma, Shanghai, China). The amount of $40-100 \mathrm{nM}$ of siRNA was introduced into cells using Hieff TransTM Liposomal transfection reagent following the manufacturer's protocol. Cells were harvested 48-72 h after transfection for immunoblotting (IB) or reverse transcription quantitative real-time (RT-qPCR). The sequence of the siRNA used here was GUAAUCUACUGGGACGGAA and as previously described ${ }^{12}$.

\section{CRISPR/Cas9-mediated gene editing}

The CRISPR/Cas9 targeting vector lentiCRISPR v2 was purchased from Addgene (Cambridge, MA, USA). The 
single guide RNA (sgRNA) for Bcl-XL was designed at https://www.benchling.com/crispr/, and the sequence of the high-scored sgRNA was 5'-CCTTGGATCCAGGAGAACGGCGG-3'. For sgRNA subcloning, the lentiCRISPR v2 vector was digested with BsmBI and ligated with BsmBI compatible annealed oligoes. The lentiviruses were generated as described ${ }^{29}$.

\section{Reverse transcription and quantitative real-time PCR}

RNA was isolated from cells using RNAiso Plus following the manufacturer's protocol (Takara, Dalian, Liaoning, China). Total RNAs of $0.5-1 \mu \mathrm{g}$ were used as templates for the RT using the PrimeScriptTM RT Reagent Kit with genomic DNA Eraser (Takara). qPCR was conducted using TB GreenTM Premix Ex TaqTM (Tli RNaseH Plus) according to the manufacturer's protocol (Takara). The primers used for qPCR were 5'-A CCATTGTGGACACACCAGG-3' and 5'-GAACCTG TGACCACCTGCTA-3' for human ARTS and 5'-CAG GGCAGGGCTACCACTAG-3' and 5'-TGATGCAGGGC CTTCATGA-3' for mouse ARTS. The primers for human and mouse p21 were previously described ${ }^{30,31}$.

\section{Immunoblotting}

Cells were harvested and lysed in lysis buffer consisting of $50 \mathrm{mM}$ Tris/ $\mathrm{HCl}$ (pH7.5), 0.5\% Nonidet P-40 (NP-40), $1 \mathrm{mM}$ EDTA, $150 \mathrm{mM} \mathrm{NaCl}, 1 \mathrm{mM}$ dithiothreitol (DTT), $0.2 \mathrm{mM}$ phenylmethylsulfonyl fluoride, $10 \mu \mathrm{M}$ pepstatin $\mathrm{A}$, and $1 \mu \mathrm{g} / \mathrm{ml}$ leupeptin. Equal amounts of clear cell lysate $(20-80 \mu \mathrm{g})$ were used for IB analysis as described previously $^{28}$.

\section{Immunoprecipitation (IP)}

IP was conducted using antibodies as indicated in the figure legends. Briefly, $500-1000 \mu \mathrm{g}$ of proteins were incubated with the indicated antibody at $4{ }^{\circ} \mathrm{C}$ for $4 \mathrm{~h}$ or overnight. Protein A or G beads (Santa Cruz Biotechnology) were then added, and the mixture was incubated at $4{ }^{\circ} \mathrm{C}$ for additional $1-2 \mathrm{~h}$. Beads were washed at least three times with lysis buffer. Bound proteins were detected by IB with antibodies as indicated in the figure legends.

\section{Luciferase reporter assay}

Cells were transfected with pGL3-RE1, RE2, or RE3 plasmid together with pGMLR-TK and the p53-encoding plasmid or the pcDNA vector as indicated in the figure. Dual-Luciferase Reporter Assay System was used to determine luciferase activity according to the manufacturer's instruction (Promega, Madison, WI, USA).

\section{Chromatin immunoprecipitation (ChIP)}

ChIP assay was performed using antibodies as indicated in the figure legends and described previously ${ }^{32}$. The reverse cross-linked immunoprecipitated DNA fragments were purified using the GeneJET Gel Extraction Kit (Thermo Scientific, Waltham, MA, USA) followed by PCR analysis for the p53-responsive DNA elements on the human ARTS promoter using the following primers 5'-G TATTAGACCCTGCCTCCATCA-3' and 5'-GAAGACT GACTTTGAGCCATCC-3'.

\section{Subcellular fractionation}

Cell suspension in the fractionation buffer $(20 \mathrm{mM}$ HEPES (pH 7.4), $10 \mathrm{mM} \mathrm{KCl,} 2 \mathrm{mM} \mathrm{MgCl} 2,1 \mathrm{mM}$ EDTA, $1 \mathrm{mM}$ EGTA, $1 \mathrm{mM}$ DTT, and protease inhibitors) was incubated for $15 \mathrm{~min}$ on ice and went through a 27 -gauge needle 10 times. After incubation on ice for another $20 \mathrm{~min}$, the samples were centrifuged at $3000 \mathrm{rpm}$ for $5 \mathrm{~min}$. The pellets contained nuclei. Supernatants were centrifuged at $8000 \mathrm{rpm}$ for $5 \mathrm{~min}$. Pellets contained mitochondria and supernatants contained the cytoplasm and membrane fraction.

\section{$\mathrm{\gamma}$-irradiation of mice}

$\mathrm{p} 53^{+/+}$and $\mathrm{p} 53^{-1-}$ mice of 8 weeks of age were subjected to whole-body $\gamma$-irradiation (5 Gy) at a dose rate of $0.75 \mathrm{~Gy} / \mathrm{min}$. Mice were sacrificed and their spleens and thymuses were harvested 0 or $6 \mathrm{~h}$ post-irradiation ${ }^{33}$. The tissues were analyzed by IB and RT-qPCR for p53, p21, and ARTS expression.

\section{Flow cytometric analysis}

The PE Annexin V Apoptosis Detection Kit I (BD Biosciences, San Diego, CA, USA) was used for apoptosis analysis according to the manufacturer's instruction. Briefly, cells were washed twice with cold phosphate-buffered saline and then re-suspended in Annexin V Binding Buffer at a concentration of $1 \times 10^{6}$ cells $/ \mathrm{ml}$. Cells were incubated with $\mathrm{PE}$ Annexin $\mathrm{V}$ and 7 -aminoactinomycin $\mathrm{D}$ for $15 \mathrm{~min}$ at room temperature in the dark. Flow cytometry was performed using a FC500 MPL flow cytometer (Beckham coulter, Indianapolis, IN, USA) within $1 \mathrm{~h}$.

\section{Cell viability assay}

The Cell Counting Kit-8 (Dojindo Molecular Technologies, Japan) was used according to the manufacturer's instructions. Cells of 2000-5000 were seeded per well in 96-well culture plates at $12 \mathrm{~h}$ post-transfection. Cell viability was determined by adding WST-8 at a final concentration of $10 \%$ to each well, and the absorbance of the samples was measured at $450 \mathrm{~nm}$ using a Microplate Reader every $24 \mathrm{~h}$ for $4-5$ days.

\section{Statistics}

All in vitro experiments were performed in biological triplicate. The Student's $t$ test or one-way analysis of variance was performed to evaluate the differences 
between two groups or more than two groups. The variance between the groups that are being statistically compared is similar. $p<0.05$ was considered statistically significant, and asterisks represent significance in the following way: ${ }^{*} p<0.05$; ${ }^{* *} p<0.01$. Quantitative data are presented as mean $\pm \mathrm{SD}$.

\section{Results}

\section{ARTS expression is induced by $\mathrm{p} 53$ in cancer cells}

Through a primary screen for p53-responsive genes by the p53-inducing agent INZ ${ }^{26,27}$, we identified ARTS as a possible p53 target gene. To confirm this, we ectopically expressed p53 in H460 lung cancer cells and indeed found that the expression of ARTS is elevated at both mRNA (Fig. 1A) and protein levels (Fig. 1B). Also, we showed that exogenous p53 induces ARTS mRNA (Fig. 1C) and protein expression (Fig. 1D) in HCT116 ${ }^{\mathrm{p} 53+/+}$ colon cancer cells. To verify these observations, several p53inducing agents were used to test whether ARTS expression is responsive to p53 activation. As shown in Fig. 1E, F, 5-fluorouracil (5-FU), Doxorubicin (DOX), or INZ treatment dramatically stimulated both mRNA and protein expression of ARTS in H460 cells. Consistently, ARTS expression could be induced by 5 -FU and DOX in two wild-type p53-harboring melanoma cell lines, SKMEL-147 and SK-MEL-103 (Fig. 1G, H). In addition, we found that ARTS expression can also be elevated upon oxidative stress triggered by $\mathrm{H}_{2} \mathrm{O}_{2}$ (Fig. 1I) that was shown to activate p53 (refs. ${ }^{34,35}$ ). The induction of ARTS expression observed could be specifically owing to p53 activation, given that the p53 target gene p21 was simultaneously induced under the same conditions (Fig.
A

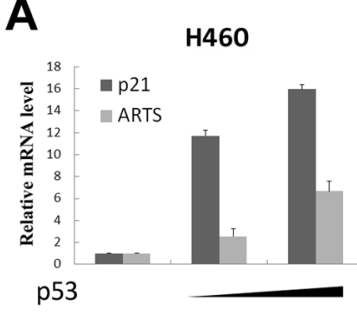

$E$

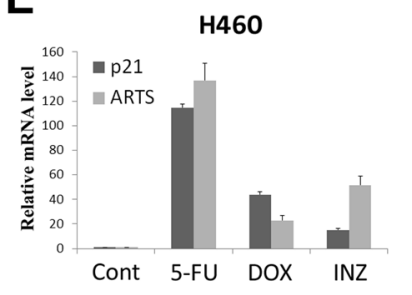

I

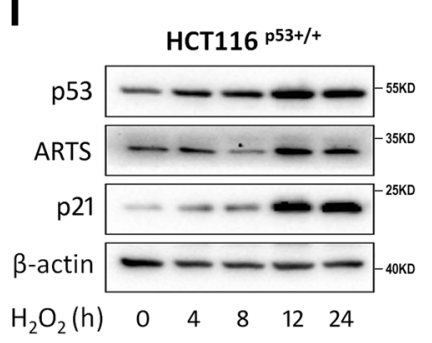

B

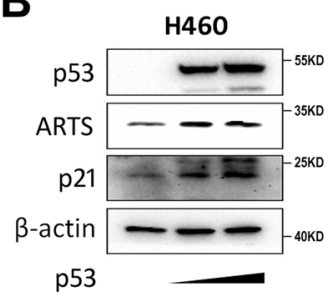

F

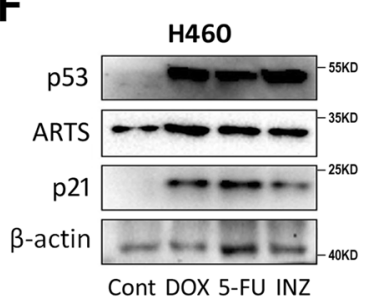

C

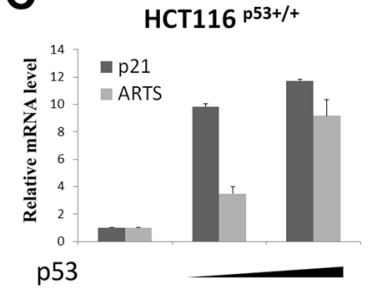

G
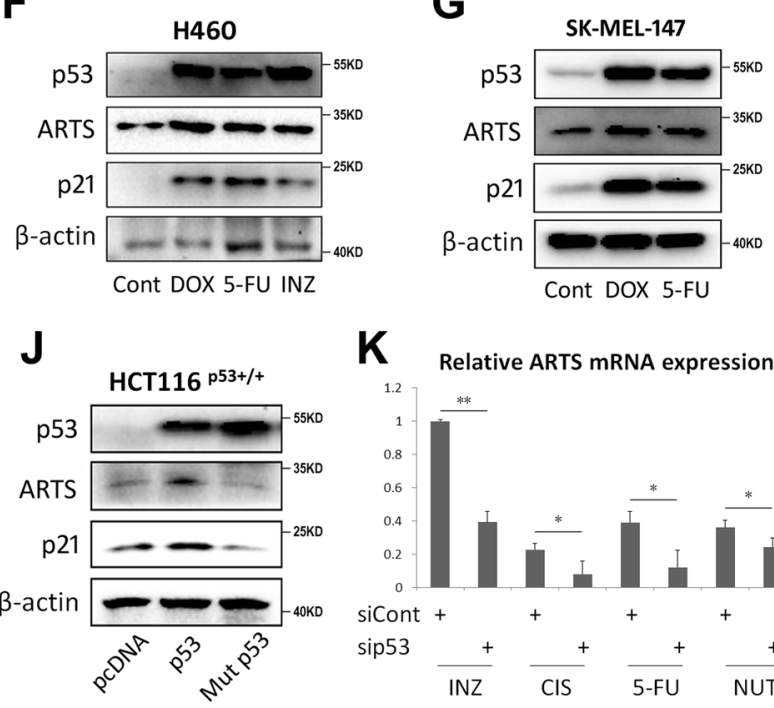

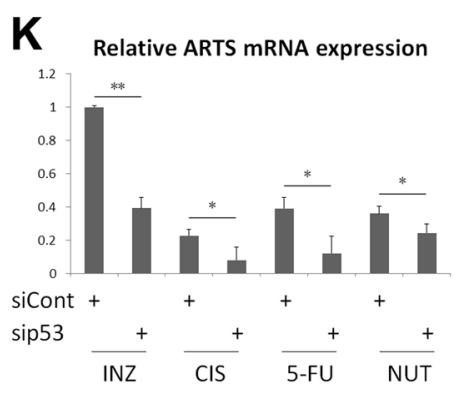

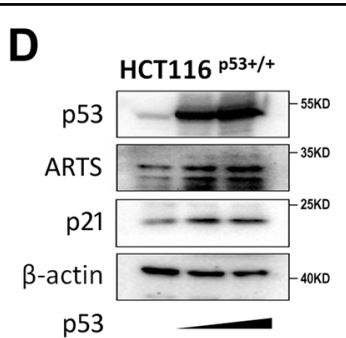

H
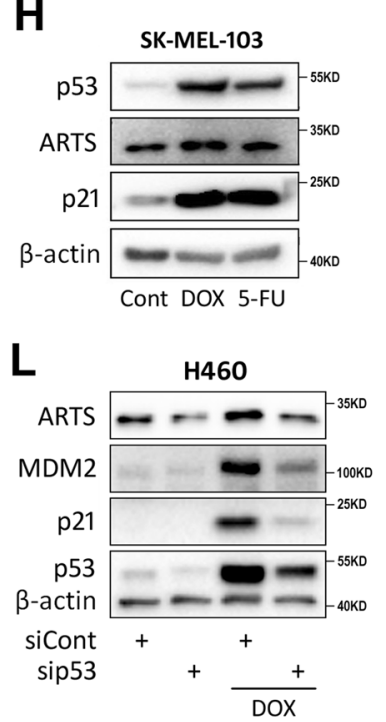

Fig. 1 p53 induces ARTS expression in cancer cells. A, B Ectopic p53 induces ARTS mRNA (A) and protein (B) expression in H460 cells. Cells were transfected with the vector or increased doses of p53 plasmid followed by RT-qPCR or IB analysis. C, D Ectopic p53 induces ARTS mRNA (C) and protein (D) expression in HCT116 ${ }^{\text {p53+/+ }}$ cells. Cells were transfected with the vector or increased doses of p53 plasmid followed by RT-qPCR or IB analysis. E, F The p53-inducing agents elevate ARTS mRNA (E) and protein (F) levels in H460 cells. Cells were treated with 5 -fluorouracil (10 $\mu$ M), Doxorubicin $(1 \mu \mathrm{M})$, or Inauhzin $(2 \mu \mathrm{M})$ for $18 \mathrm{~h}$ followed by RT-qPCR or IB analysis. G, $\mathbf{H}$ The p53-inducing agents elevate ARTS expression in melanoma cell lines. Cells were treated with 5 -fluorouracil $(10 \mu \mathrm{M})$ or Doxorubicin $(1 \mu \mathrm{M})$ for $18 \mathrm{~h}$ followed by IB analysis. I $\mathrm{H}_{2} \mathrm{O}_{2}$-induced oxidative stress increases ARTS expression. Cells were treated with $\mathrm{H}_{2} \mathrm{O}_{2}$ for the indicated time followed by IB analysis. J Wild-type p53, but not mutant p53$\mathrm{R} 175 \mathrm{H}$, induces ARTS expression. Cells were transfected with plasmids as indicated followed by IB analysis. K Ablation of p53 reduces ARTS mRNA expression upon multiple stress conditions. Cells were transfected with p53 siRNA and treated with Inauhzin (2 $\mu \mathrm{M})$, Cisplatin (10 $\mu \mathrm{M})$, 5 -fluorouracil $(10 \mu \mathrm{M})$, or Nutlin-3 $(20 \mu \mathrm{M})$ for $18 \mathrm{~h}$ before harvest for RT-qPCR analysis. L Ablation of p53 reduces ARTS protein expression upon DNA damage stress. Cells were transfected with p53 siRNA and treated with Doxorubicin $18 \mathrm{~h}$ before harvest for IB analysis. 
$1 \mathrm{~A}-\mathrm{I})$ and that ectopic mutant $\mathrm{p} 53-\mathrm{R} 175 \mathrm{H}$ exerted no effect on ARTS or p21 expression (Fig. 1J). Then we determined whether endogenous p53 is required for ARTS induction upon different stress signals. ARTS mRNA levels were assessed in HCT11 $6^{\mathrm{p} 53+/+}$ cells treated with INZ, Cisplatin, 5-FU, or Nutlin-3 following knockdown of p53. As expected, the expression of ARTS in response to these treatments was significantly reduced upon p53 depletion (Fig. 1K), which is further validated by the protein expression of ARTS in H460 cells under both normal and DNA damage conditions (Fig. 1L). Therefore, these results indicate that the Sept4/ARTS gene is a p53inducible gene in response to various stress signals in cancer cells.

\section{$\gamma$-irradiation induces ARTS expression dependent on p53 in mice}

Since ARTS is required for tumor suppression in vivo ${ }^{17,23}$, we tested whether this tumor suppressor can be activated through $\mathrm{p} 53$ in mice. The $\mathrm{p} 53^{+/+}$and $\mathrm{p} 53^{-/-}$mice were exposed to $\gamma$-irradiation, and the radiosensitive organs, thymuses and spleens ${ }^{33}$, were harvested for analysis of the expression of murine p53, ARTS, and p21. As shown in Fig. $2 \mathrm{~A}, \gamma$-irradiation drastically boosted the protein levels of $\mathrm{p} 53$ and ARTS in the thymuses of the $\mathrm{p} 53^{+/+}$mice but not in those of the $\mathrm{p} 53^{-1-}$ mice. The induction of ARTS in response to $\gamma$-irradiation could be due to the increased transcriptional activity of p53, as evidenced by the upregulation of its mRNA level (Fig. 2B). In line with these data, the irradiated spleens also displayed higher expression of murine ARTS in a p53-dependent fashion (Fig. 2C, D). Together with the results in Fig. 1, these findings demonstrate that p53 induces ARTS expression in response to various stressors not only in cancer cells but also in healthy cells in mice.

\section{p53 transcriptionally activates ARTS expression by associating with its promoter}

Since p53 mainly functions as a transcription factor, we speculated that p53 may enhance Sept4/ARTS gene transcription by binding to its promoter. Indeed, by carefully analyzing the genomic sequence of the human Sept4/ARTS gene using p53MH algorithm ${ }^{36}$, we found three potential p53-responsive elements (p53-REs) located at -3087 and $-2279 \mathrm{bp}$ before the transcription-start site and +9720 bp within the intron of the Sept4/ARTS gene (Fig. 3A). To determine whether p53 activates transcription of ARTS through any of these REs, we tested the luciferase reporter gene expression driven by each of the REs. Remarkably, p53 induced luciferase activity only via the p53-RE2 but not RE1 or RE3 (Fig. 3B). We then examined whether p53 binds to the Sept4/ARTS gene promoter at the p53-RE2 site by performing a ChIP assay.
A

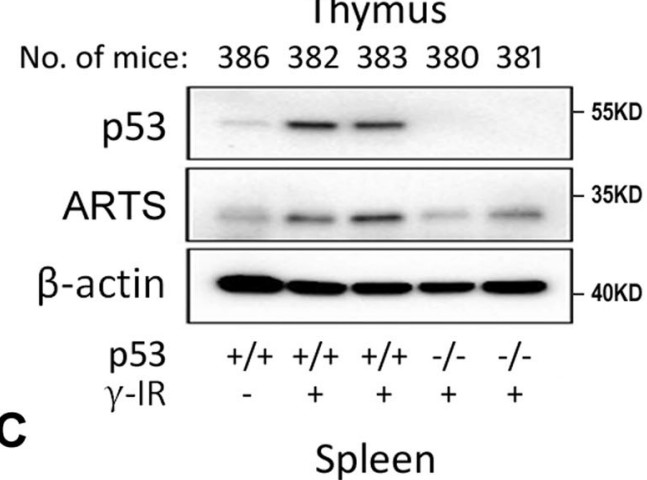

No. of mice: 386382383380381

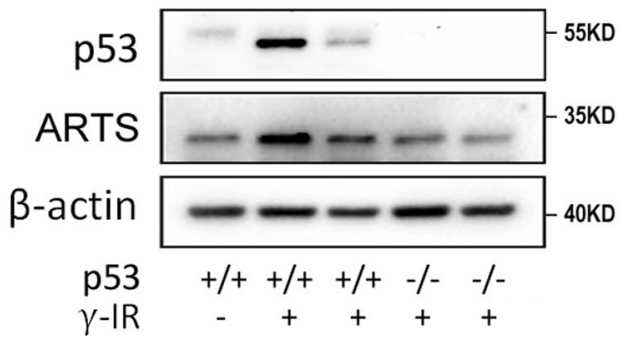

B
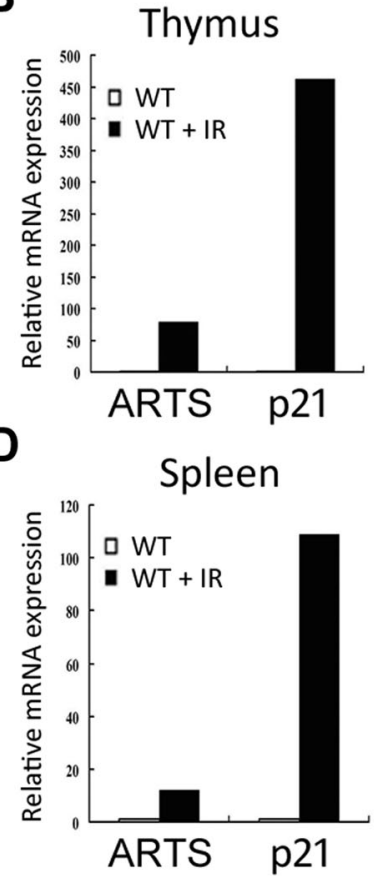

Fig. 2 ARTS expression is induced by $\mathbf{p}$-irradiation through $\mathbf{p 5 3}$ in mice. A, B ARTS mRNA (A) and protein (B) expression is elevated in the irradiated murine thymuses. The irradiated mice were sacrificed, and the thymuses were freshly harvested and subjected to IB or RT-qPCR analysis. $\mathbf{C}$, D ARTS mRNA $(\mathbf{C})$ and protein (D) expression is elevated in the irradiated murine spleens. The irradiated mice were sacrificed, and the spleens were freshly harvested and subjected to IB or RT-qPCR analysis. 
A

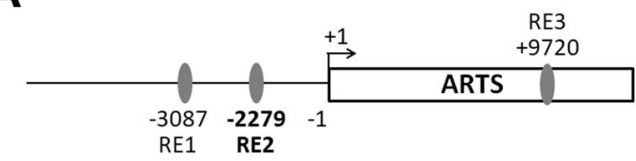

p53 consensus: RRRCWWGYYY

RE1: GACCTTGTTG

RE2: GGACTTGTGC

RE3: AAGCTAGTAT

\section{B}

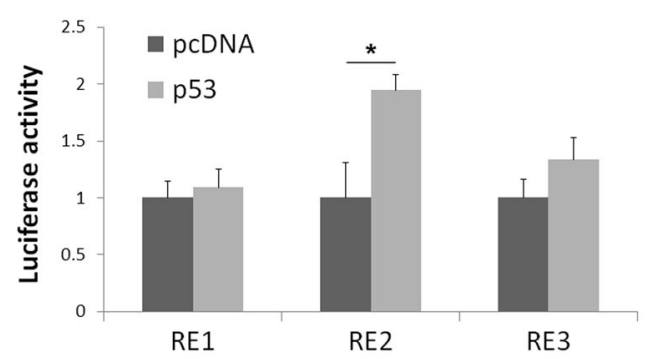

C

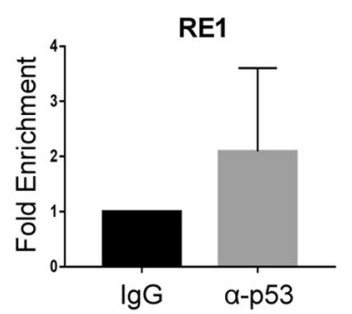

D

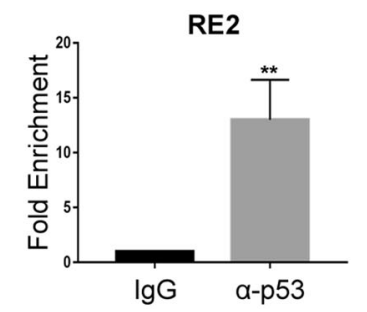

Fig. 3 p53 associates and activates ARTS promoter. A A schematic of the predicted p53-responsive elements on the promoter or within the intron of ARTS. B p53 induces the expression of a luciferase reporter gene driven by RE1. H1299 cells were transfected with plasmids as indicated in the "Materials and methods" for over $30 \mathrm{~h}$ followed by the luciferase assay. C, D p53 associates with RE2, but not RE1, on the ARTS promoter. $\mathrm{HCT} 116^{\mathrm{p} 53+/+}$ cells were prepared for the ChIP-qPCR analysis using the antibodies as indicated.

Consistently, p53 markedly associated with the promoter fragment harboring the RE2 but not RE1 (Fig. 3C, D). Taken together, these results demonstrate that ARTS is a bona fide p53 target gene.

\section{ARTS binds to p53 without affecting p53 protein stability}

ARTS has been documented as a key pro-apoptotic protein $^{14,15}$ acting by targeting XIAP (refs. ${ }^{15,19,20}$ ) and Bcl-2 (ref. ${ }^{21}$ ). Thus we sought to explore whether ARTS plays a role in $\mathrm{p} 53$-associated apoptotic pathway, since it is a p53-inducible gene. Intriguingly, through our recent work by screening mutant p53-interacting proteins in ovarian cancer as previously described ${ }^{29}$, we unexpectedly found that mutant p53 may interact with a peptide (KLQDQALKE) encoded by the SEPT4 gene through a co-immunoprecipitation (co-IP) assay coupled with mass spectrometry (MS) analysis (Fig. 4A). This observation prompted us to test whether ARTS binds to wild-type p53 as well, because both wild-type and mutant p53 share common binding partners in many cases, such as MDM2 and TRIM71 (refs. ${ }^{29,37}$ ). By co-expressing exogenous p53 and Flag-ARTS in H1299 cells followed by co-IP-IB assays, we found that exogenous p53 could be coimmunoprecipitated with Flag-ARTS using an anti-Flag antibody (Fig. 4B). Also, Flag-ARTS was coimmunoprecipitated with exogenous p53 using an antip53 antibody (Fig. 4C). Furthermore, the interaction between endogenous ARTS and p53 proteins was verified by a co-IP assay using an anti-ARTS antibody in H460 cells (Fig. 4D). Since some of the p53-inducible proteins, such as NGFR (ref. ${ }^{12}$ ) and PHLDB3 (ref. ${ }^{13}$ ) identified by the same microarray screening as mentioned above ${ }^{26}$, can promote $\mathrm{p} 53$ protein turnover by binding to the latter, we tested whether ARTS can do so as well. However, transient overexpression of ARTS seemed not to affect the protein level of either exogenous or endogenous p53 in H1299 and HCT116 ${ }^{\mathrm{p} 53+/+}$ cells (Fig. 4E, F). Thus these results demonstrated that ARTS interacts with p53 in cancer cells and also suggested that ARTS may regulate p53 activity through their interaction.

\section{Ectopic ARTS enhances p53-Bcl-XL interaction by sequestering $\mathrm{p} 53$ in the mitochondria}

Although the cytosolic p53 induces apoptosis directly through the mitochondrial pathway ${ }^{3}$, the mechanism that relocates p53 to the mitochondria remains to be determined. Given that ARTS was shown to reside at the outer membrane of the mitochondria at the initiating stage of apoptosis $^{15}$, we examined whether ARTS is responsible for the mitochondrial localization of p53. Through fractionation of cellular components, we observed that ectopically expressed Flag-ARTS predominantly locates at the mitochondria, and ARTS markedly increases the mitochondrial fraction of endogenous p53 (Fig. 5A). The mitochondrial fraction was also used for co-IP analysis. Consistently, we verified the interaction of endogenous 
A

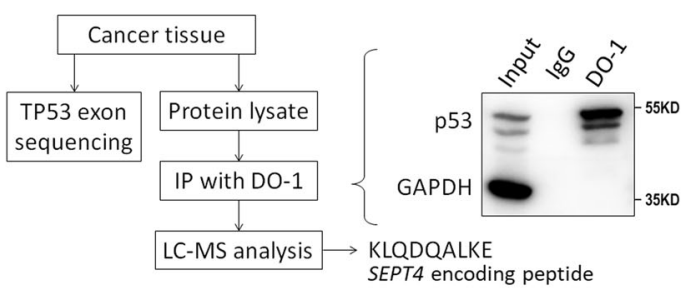

B
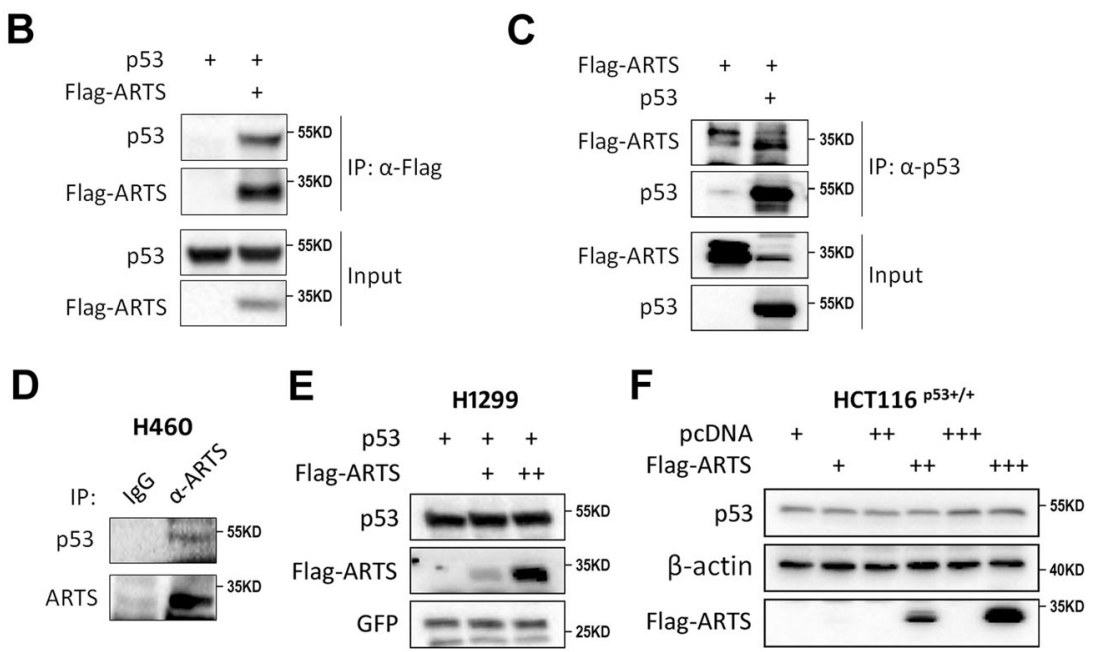

Fig. 4 ARTS interacts with p53. A A schematic of screening for p53-interacting proteins in the cancer tissue harboring mutant p53-S241F. B, C The exogenous interactions between ARTS and p53. H1299 cells were transfected with plasmids as indicated followed by co-IP assays using the anti-flag (B) or anti-p53 (C) antibody. The bound complexes were assessed by IB analysis. D The endogenous interaction between ARTS and p53. The H460 cell lysates were prepared for co-IP-IB assays using the antibodies as indicated. E, F Ectopic ARTS does not affect exogenous (E) and endogenous $(\mathbf{F})$ p53 stability. $\mathrm{H} 1299$ or $\mathrm{HCT} 116^{\mathrm{p} 53+/+}$ cells were transfected with plasmids as indicated followed by IB analysis.

ARTS and p53 in the mitochondria (Figs. 5B and S1). It was noted that DOX or Etoposide treatment does not significantly enhance the ARTS-p53 interaction (Figs. 5B and S1). This was probably because ARTS might recruit p53 to the mitochondria through a "hit-and-run" mechanism and it would translocate from the mitochondria to the cytoplasm and the nucleus in the later stage of apoptosis ${ }^{14}$. Then we sought to determine whether ARTS regulates p53's function during mitochondrial apoptosis. In the early stage of apoptosis, as p53 binds to Bcl-XL, Bcl-2, and BAK (ref. ${ }^{3}$ ), we thus tested whether ARTS regulates their interactions at the mitochondria. Interestingly, as shown in Fig. $5 \mathrm{C}$, p53 could bind to Bcl$\mathrm{XL}$ as reported previously ${ }^{4,5}$, and this interaction was markedly increased when ARTS was co-expressed in cells. Conversely, knockdown of ARTS significantly reduced the p53-Bcl-XL interaction (Fig. 5D). Therefore, these findings demonstrate that ARTS sequesters p53 in the mitochondria and consequently enhances the interaction of p53 with Bcl-XL in this subcellular compartment and also suggest that ARTS may collaborate with p53 in triggering apoptosis, which will be addressed as follows.

\section{The interplay of p53 and ARTS promotes apoptosis in cooperation}

To determine the biological significance of the p53ARTS cascade, we assessed whether ARTS is involved in p53-induced apoptosis by flow cytometric analysis. Ectopic ARTS marginally induced apoptosis under the unstressed condition (Figs. 6A, B and S2A, B), which is in line with the former studies ${ }^{19,20}$. Interestingly, Cisplatin or Nutlin-3 treatment significantly sensitized HCT116 $6^{\mathrm{p} 53+/+}$ (Fig. 6A, B) and H460 cells (Fig. S2A, B) to apoptosis induced by ectopic ARTS, suggesting that ARTS may collaborate with activated p53 to prompt apoptosis under stress conditions. Additionally, we examined whether endogenous ARTS is required for stress-triggered apoptosis. As illustrated in Fig. 6C, D, knockdown of ARTS significantly, though moderately, impaired DNA damageinduced apoptosis. Of note, we showed that ARTS depletion dramatically represses Nutlin-3-induced apoptosis to a greater extent than Cisplatin-induced apoptosis in H460 cells (Fig. 6E, F). Considering that Cisplatin triggers apoptosis through genotoxic stress that might be partially p53 independent, while Nutlin-3 is a specific p53 

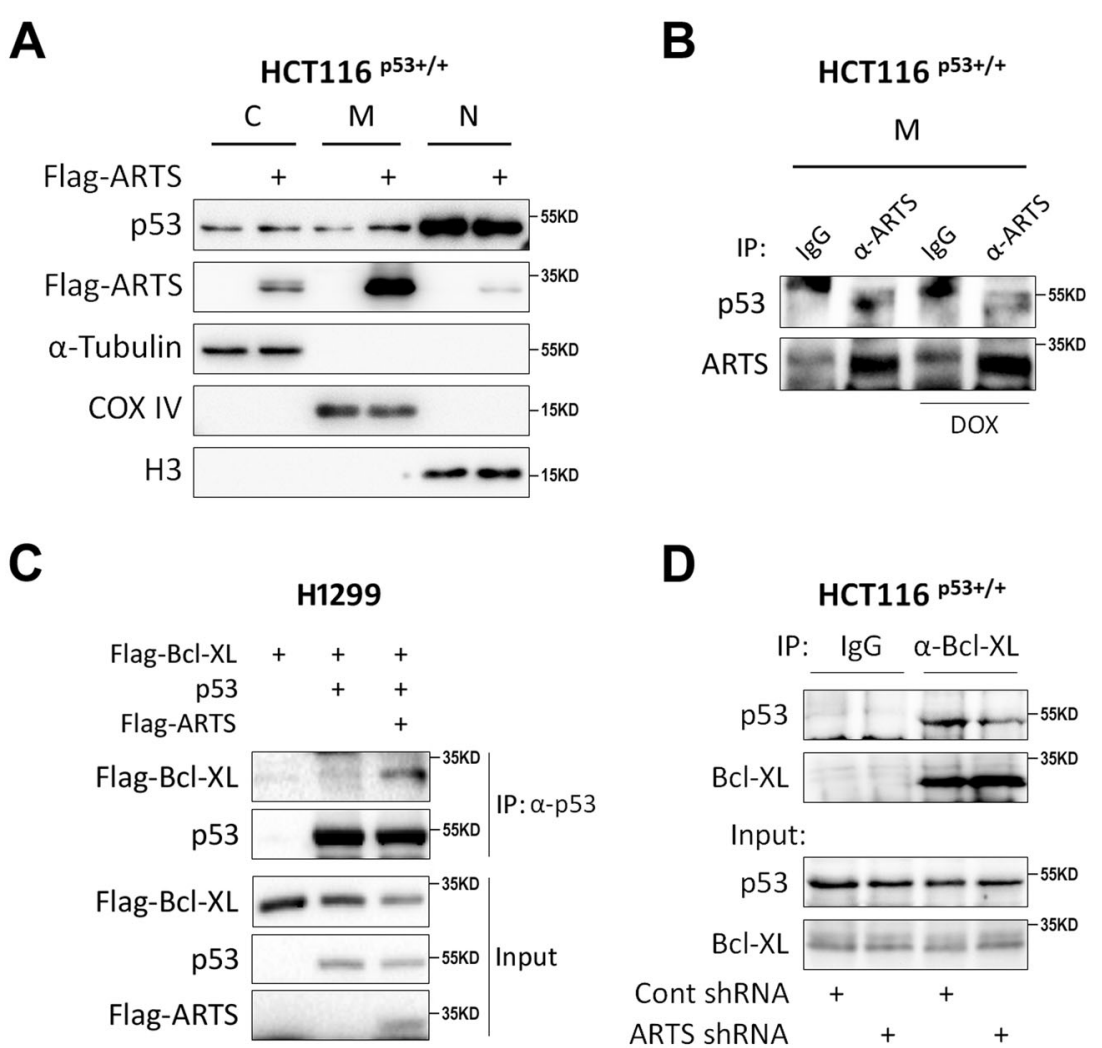

Fig. 5 ARTS enhances p53-Bcl-XL interaction. A Overexpression of ARTS promotes mitochondrial accumulation of p53. HCT116 $6^{\mathrm{p} 53+/+}$ cells were transfected with Flag-ARTS or the vector plasmid and subjected to cellular component fractionation. IB assays were performed to assess the expression of p53 and ARTS in the nucleus, cytoplasm, and mitochondria, respectively. The expression of COX IV indicates the mitochondrial fraction. B ARTS interacts with $\mathrm{p} 53$ in the mitochondria. The mitochondrial fraction of HCT116 $6^{\mathrm{p} 53+/+}$ cells was prepared for the co-IP assay using antibodies as indicated. C Overexpression of ARTS enhances the interaction of p53 with BCl-XL. H1299 cells were transfected with plasmids as indicated followed by the co-IP-IB analyses. D Knockdown of ARTS reduces the interaction of p53 with BCl-XL. HCT116 $6^{\mathrm{p} 53+/+}$ cells expressing control or ARTS shRNA were subjected to co-IP-IB analyses using antibodies as indicated.

agonist inducing p53-dependent apoptosis, we believed that endogenous ARTS is more selectively responsible for p53-induced apoptosis. The results were also verified in ARTS-depleted HCT116 ${ }^{\mathrm{p} 53+/+}$ cells (Fig. S2C, D). Interestingly, we found that the 553 levels in the mitochondria increase upon Cisplatin or Nutlin-3 treatment (Fig. S3A), suggesting a direct role of p53 in the mitochondrial apoptosis. In line with the result above (Fig. 5A), ARTS depletion reduced p53 levels in the mitochondria under the apoptotic condition (Fig. S3A). Furthermore, we asked whether Bcl-XL is involved in ARTS-induced apoptosis, given that ARTS enhances the interaction between p53 and Bcl-XL (Fig. 5C, D). The Bcl-XL-knockout HCT116 $6^{\mathrm{p} 53+/+}$ cell line generated via the CRISPR-Cas9 method was employed in the study (Fig. S3B). As expected, knockout of Bcl-XL markedly induced the level of cleaved PARP (Fig. S3B) and apoptosis (Fig. 6G, H). Remarkably, Bcl-XL depletion abrogated ARTS-induced apoptosis in response to p53 activation in $\mathrm{HCT} 116^{\mathrm{p} 53+/+}$ cells (Fig. 6G, H). Altogether, these results demonstrate that ARTS promotes apoptosis in cooperation with p53.

\section{Discussion}

The tumor-suppressor p53 promotes cancer cell death through transcriptional activation of multiple proapoptotic genes or direct interaction with $\mathrm{Bcl}-2$ family proteins in the mitochondria. Herein we have unveiled ARTS as a novel transcriptional target and a positive regulator of p53 during mitochondrial apoptosis (Fig. 7). We showed that p53 transcriptionally induces ARTS expression in cancer cells and in mice (Figs. 1 and 2) by binding to the ARTS promoter (Fig. 3). In addition, ARTS interacts with and detains p53 in the mitochondria, resulting in increased interaction between p53 and Bcl-XL (Figs. 4 and 5) and augmented apoptosis (Fig. 6). Thus our study demonstrates that ARTS plays a critical role in the p53-induced mitochondrial apoptotic pathway. 
A

B
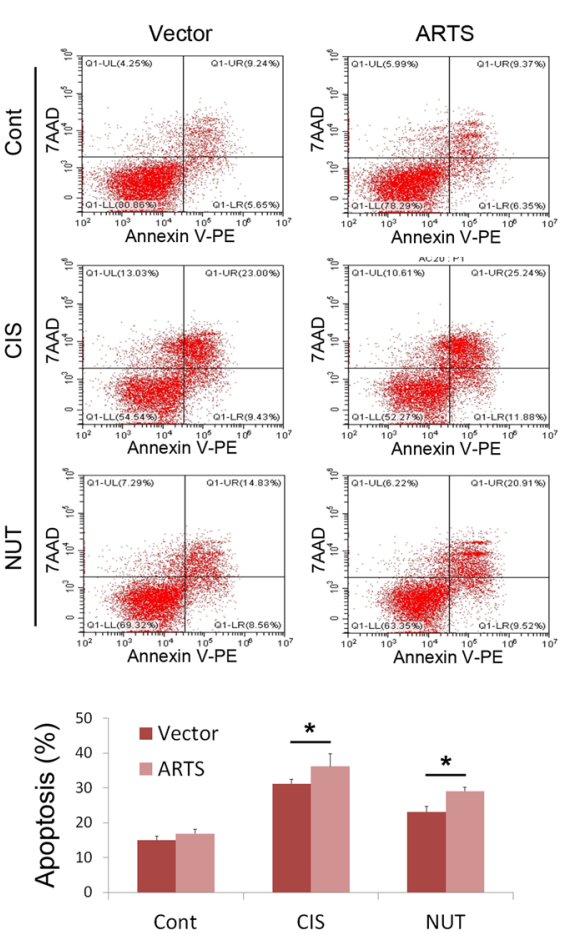

E

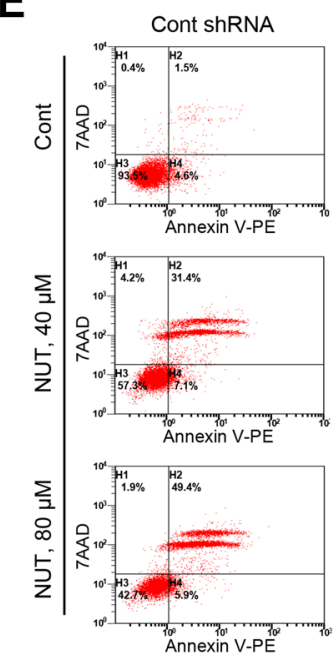

F
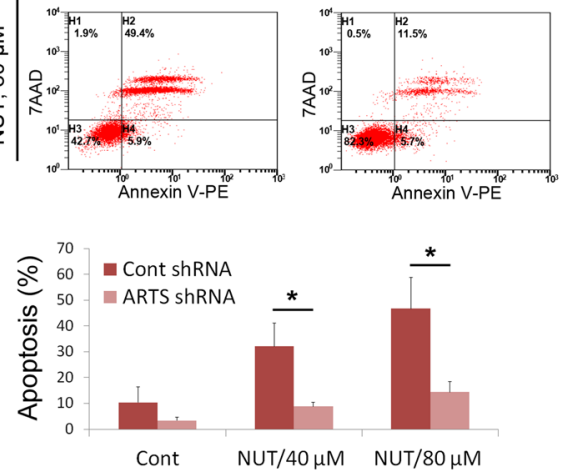

C
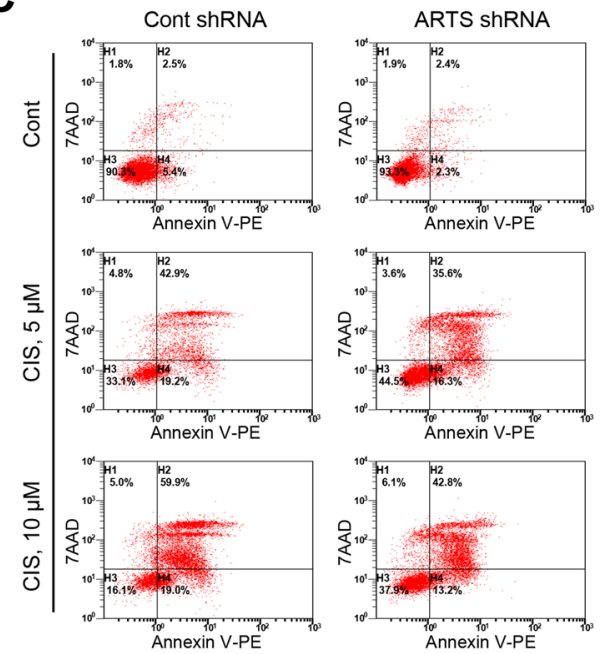

D

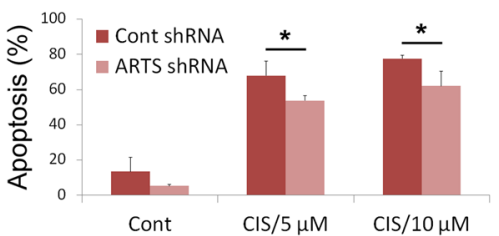

G
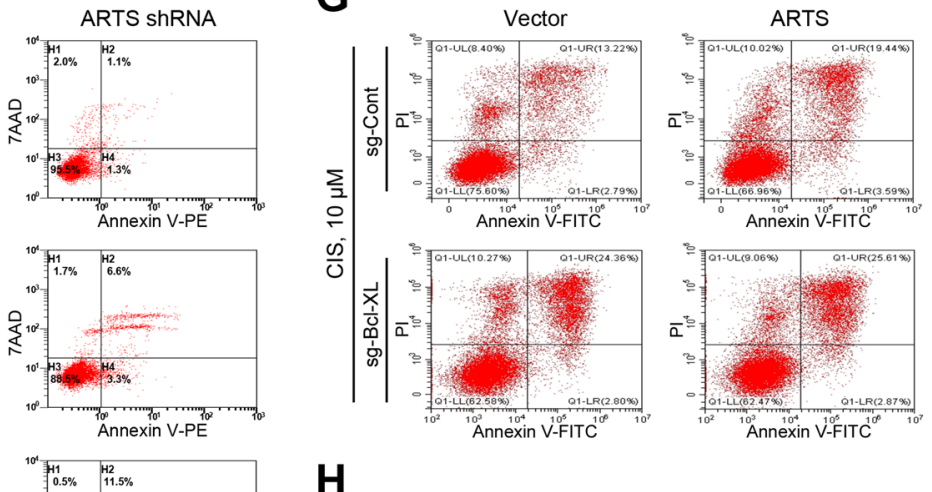

H

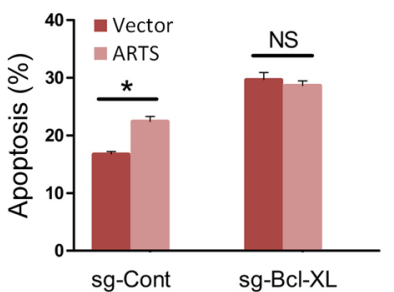

Fig. 6 ARTS promotes apoptosis in cooperation with p53. A, B Overexpression of ARTS significantly augments Cisplatin- or Nutlin-3-induced apoptosis. HCT1 16 ${ }^{\mathrm{p} 53+/+}$ cells stably overexpressing the vector or ARTS were treated with $20 \mu \mathrm{M}$ Cisplatin or $10 \mu \mathrm{M}$ Nutlin-3 for $48 \mathrm{~h}$. The apoptosis of the treated and untreated control cells were then analyzed by flow cytometry. C, D Ablation of ARTS diminishes Cisplatin-induced apoptosis. H460 cells stably expressing control or ARTS shRNA were treated with 0, 5, or $10 \mu \mathrm{M}$ Cisplatin for $48 \mathrm{~h}$ and subjected to flow cytometric analysis for apoptosis. E, F Ablation of ARTS markedly impairs p53-induced apoptosis. H460 cells stably expressing control or ARTS shRNA were treated with 0, 40, or $80 \mu \mathrm{M}$ Nutlin-3 for $48 \mathrm{~h}$ and subjected to flow cytometric analysis for apoptosis. G, H Depletion of Bcl-XL impairs ARTS-induced apoptosis in response to Cisplatin treatment. The control and BCl-XL-knockout HCT116 ${ }^{\mathrm{p} 53+/+}$ cells were transfected with the control vector or ARTS-expressing plasmid and treated with Cisplatin for $48 \mathrm{~h}$, followed by flow cytometric analysis for apoptosis. 


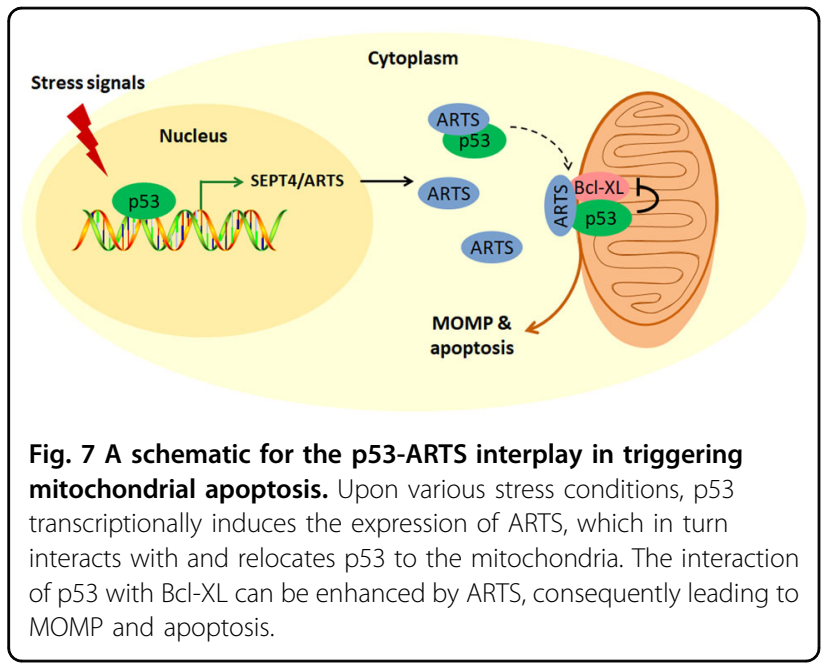

To keep ARTS expression at an appropriate level is vital for cell survival, while increased expression of ARTS provokes cell death upon various stimuli. It was shown that the ARTS protein level is strictly monitored by the proteasome degradation system $^{18}$. The E3-ubiquitin ligase, Parkin, specifically binds to ARTS and induces its ubiquitination and degradation ${ }^{38}$. Additionally, XIAP also serves as an E3-ubiquitin ligase targeting ARTS for degradation constituting a negative feedback loop ${ }^{39}$. However, the mechanism for the Sept4/ARTS gene transcription remains unclear. Our study has revealed that DNA damage stress triggered by 5-FU, DOX, or $\gamma$-irradiation elevates ARTS mRNA level in cancer cells (Fig. $1 \mathrm{E}-\mathrm{G}, \mathrm{L}$ ) and in vivo (Fig. 2), which is consistent with a previous study showing that treatment of SH-SY5Y cells with Etoposide upregulates ARTS expression ${ }^{38}$. More importantly, we found that the upregulation of ARTS is dependent on p53 activation, because ectopic expression of p53 increases, while knockdown of p53 reduces both mRNA and protein levels of ARTS (Fig. 1A-D, J-L). Moreover, p53 can associate with the ARTS promoter (Fig. 3D) and enhance its ability to drive the expression of a luciferase reporter gene (Fig. 3B). Collectively, these findings explicitly demonstrate for the first time the mechanism underlying the Sept4/ARTS gene transcription by $\mathrm{p} 53$.

It was unexpected but interesting to notice that the SEPT4-encoding proteins might bind to mutant p53Y220C through a co-IP-MS analysis in our recent study ${ }^{29}$. The TP53 gene is thus far the most frequently mutated tumor suppressor in human cancer. The p53-Y220C mutant was shown to not only lose the tumor-suppressive activity but also be able to boost cancer cell proliferation and migration ${ }^{29}$. Although the function of wild-type and mutant p53s diverges in cancer development, they do usually share common interacting partners as described in previous studies ${ }^{40}$. We therefore speculated that ARTS may interact with wild-type p53 and convincingly testified this idea by showing the ARTS-p53 interaction through a set of co-IP assays (Figs. 4B-D, 5B, and S1). It was further found that ARTS cooperates with p53 in bolstering mitochondrial apoptosis through improvement of the p53-Bcl-XL interaction (Figs. 5C, D, 6, and S2). Therefore, we propose here an alternative mechanism for proapoptotic function of ARTS in cancer, in addition to its inhibitory effects on XIAP (refs. ${ }^{15,19,20}$ ) and Bcl-2 (ref. ${ }^{21}$ ).

Although several p53 target genes, such as PUMA, NOXA, and BAX, may be potent in executing p53's apoptotic function, we ascertained in our study that ARTS is also involved in this process. First, although ectopic ARTS has trivial effect on apoptosis of HCT116 cells culturing in the normal condition, it significantly enhances apoptosis when cells are under stress conditions with activated p53 (Figs. 6A, B and S2A, B). Additionally, depletion of endogenous ARTS significantly impairs apoptosis induced by Cisplatin or Nutlin-3 (Figs. 6C-F and $\mathrm{S} 2 \mathrm{C}, \mathrm{D})$. Intriguingly, we found that robust expression of ARTS is more essential to Nutlin-3-induced apoptosis (Fig. 6E, F). It is probably owing to the different molecular mechanisms behind Cisplatin- or Nutlin-3-induced apoptosis. Cisplatin triggers DNA damage response and induces apoptosis via various signaling pathways, including activation of p53 as one of the mechanisms, while Nutlin-3 selectively evokes p53-dependent apoptosis ${ }^{41}$. Thus our data strongly demonstrate that ARTS is particularly required for p53-induced apoptosis.

In conclusion, ARTS can be transcriptionally activated by p53 and endorse p53's mitochondrial apoptotic function by binding to $\mathrm{p} 53$ and enhancing its interaction with Bcl-XL.

\section{Acknowledgements}

We thank the laboratory members for active and helpful discussion, Yazhen Gui for assistance with laboratory work, and the innovative research team of high-level local university in Shanghai.

\section{Author details \\ ${ }^{1}$ Fudan University Shanghai Cancer Center and Institutes of Biomedical Sciences, Fudan University, Shanghai 200032, China. ${ }^{2}$ Department of Oncology, Shanghai Medical College, Fudan University, Shanghai 200032, China. \\ ${ }^{3}$ Department of Biochemistry \& Molecular Biology and Tulane Cancer Center, Tulane University School of Medicine, New Orleans, LA 70112, USA. \\ ${ }^{4}$ Department of Physiology, Medical College of Nanchang University, Nanchang 330006, China. ${ }^{5}$ Cell Death and Cancer Research Laboratory, Department of Biology, University of Haifa, Haifa 31905, Israel. ${ }^{6}$ Key Laboratory of Breast Cancer in Shanghai, Fudan University Shanghai Cancer Center, Fudan University, Shanghai 200032, China. ${ }^{7}$ Shanghai Key Laboratory of Medical Epigenetics, International Co-laboratory of Medical Epigenetics and Metabolism, Ministry of Science and Technology, Institutes of Biomedical Sciences, Fudan University, Shanghai 200032, China. ${ }^{8}$ Present address: Department of Physiology, Medical College of Nanchang University, Nanchang 330006, China}

\section{Author contributions}

Q.H. and J.C. designed, conducted, and analyzed most of the experiments under supervision of H.L. and X.Z.; J.L. prepared the irradiated mouse tissues; Y.H. conducted the ChIP assay; Y.G. conducted part of apoptosis analyses; S.L. 
provided part of reagents and helpful suggestions; S.X.Z. conducted the microarray screen and prepared part of critical materials; H.L. and X.Z. designed the experiments, analyzed the data, and composed the manuscript.

\section{Ethics statement}

The study was approved by the Ethics Committee of Fudan University Shanghai Cancer Center.

\section{Funding}

The study was supported in part by the National Natural Science Foundation of China (Nos. 81874053, 82072879, and 81702352) to X.Z. and Q.H.; Reynolds and Ryan Families Chair Fund of Translational Cancer to H.L.; and Israel Science Foundation (ISF) Grant 822/12 to S.L.

\section{Conflict of interest}

The authors declare that they have no conflict of interest.

\section{Publisher's note}

Springer Nature remains neutral with regard to jurisdictional claims in published maps and institutional affiliations.

Supplementary information The online version contains supplementary material available at https://doi.org/10.1038/s41419-021-03463-8.

Received: 9 June 2020 Revised: 13 January 2021 Accepted: 19 January 2021 Published online: 24 February 2021

\section{References}

1. Riley, T., Sontag, E., Chen, P. \& Levine, A. Transcriptional control of human p53regulated genes. Nat. Rev. Mol. Cell Biol. 9, 402-412 (2008).

2. Levine, A. J. The many faces of p53: something for everyone. J. Mol. Cell Biol. 11, 524-530 (2019).

3. Green, D. R. \& Kroemer, G. Cytoplasmic functions of the tumour suppressor p53. Nature 458, 1127-1130 (2009).

4. Mihara, M. et al. p53 has a direct apoptogenic role at the mitochondria. Mol. Cell 11, 577-590 (2003).

5. Moll, U. M., Marchenko, N. \& Zhang, X. K. p53 and Nur77/TR3 - transcription factors that directly target mitochondria for cell death induction. Oncogene $\mathbf{2 5}$ 4725-4743 (2006)

6. Leu, J. I., Dumont, P., Hafey, M., Murphy, M. E. \& George, D. L. Mitochondrial p53 activates Bak and causes disruption of a Bak-Mcl1 complex. Nat. Cell Biol. 6, 443-450 (2004)

7. Zhou, X., Cao, B. \& Lu, H. Negative auto-regulators trap p53 in their web. J. Mol. Cell Biol. 9, 62-68 (2017).

8. Liu, Y., Tavana, O. \& Gu, W. p53 modifications: exquisite decorations of the powerful guardian. J. Mol. Cell Biol. 11, 564-577 (2019).

9. Oliner, J. D. et al. Oncoprotein MDM2 conceals the activation domain of tumour suppressor p53. Nature 362, 857-860 (1993).

10. Haupt, Y., Maya, R., Kazaz, A. \& Oren, M. Mdm2 promotes the rapid degradation of p53. Nature 387, 296-p299 (1997)

11. Kubbutat, M. H., Jones, S. N. \& Vousden, K. H. Regulation of p53 stability by Mdm2. Nature 387, 299-303 (1997).

12. Zhou, X. et al. Nerve growth factor receptor negates the tumor suppressor p53 as a feedback regulator. elife 5, e15099 (2016).

13. Chao, T. et al. Pleckstrin homology domain-containing protein PHLDB3 supports cancer growth via a negative feedback loop involving p53. Nat. Commun. 7, 13755 (2016).

14. Larisch, S. et al. A novel mitochondrial septin-like protein, ARTS, mediates apoptosis dependent on its P-loop motif. Nat. Cell Biol. 2, 915-921 (2000).
15. Edison, N. et al. The IAP-antagonist ARTS initiates caspase activation upstream of cytochrome C and SMAC/Diablo. Cell Death Differ. 19 356-368 (2012).

16. Mandel-Gutfreund, Y., Kosti, I. \& Larisch, S. ARTS the unusual septin: structural and functional aspects. Biol. Chem. 392, 783-790 (2011).

17. Elhasid, R. et al. Mitochondrial pro-apoptotic ARTS protein is lost in the majority of acute lymphoblastic leukemia patients. Oncogene $\mathbf{2 3}, \mathbf{5 4 6 8 - 5 4 7 5}$ (2004).

18. Lotan, R. et al. Regulation of the proapoptotic ARTS protein by ubiquitinmediated degradation. J. Biol. Chem. 280, 25802-25810 (2005).

19. Gottfried, Y., Rotem, A., Lotan, R., Steller, H. \& Larisch, S. The mitochondrial ARTS protein promotes apoptosis through targeting XIAP. EMBO J. 23, 1627-1635 (2004).

20. Garrison, J. B. et al. ARTS and Siah collaborate in a pathway for XIAP degradation. Mol. Cell 41, 107-116 (2011).

21. Edison, N. et al. Degradation of Bcl-2 by XIAP and ARTS promotes apoptosis. Cell Rep. 21, 442-454 (2017).

22. Kissel, $\boldsymbol{H}$. et al. The Sept4 septin locus is required for sperm terminal differentiation in mice. Dev. Cell 8, 353-364 (2005).

23. Garcia-Fernandez, M. et al. Sept4/ARTS is required for stem cell apoptosis and tumor suppression. Genes Dev. 24, 2282-2293 (2010).

24. Fuchs, Y. et al. Sept4/ARTS regulates stem cell apoptosis and skin regeneration. Science 341, 286-289 (2013).

25. Koren, E. et al. ARTS mediates apoptosis and regeneration of the intestinal stem cell niche. Nat. Commun. 9, 4582 (2018).

26. Liao, J. M., Zeng, S. X., Zhou, X. \& Lu, H. Global effect of inauhzin on human p53-responsive transcriptome. PLoS ONE 7, e52172 (2012).

27. Zhang, Q. et al. A small molecule Inauhzin inhibits SIRT1 activity and suppresses tumour growth through activation of p53. EMBO Mol. Med. 4, 298-p312 (2012).

28. Zhou, X., Hao, Q., Liao, J., Zhang, Q. \& Lu, H. Ribosomal protein S14 unties the MDM2-p53 loop upon ribosomal stress. Oncogene 32, 388-396 (2013).

29. Chen, Y. et al. Ubiquitin ligase TRIM71 suppresses ovarian tumorigenesis by degrading mutant p53. Cell Death Dis. 10, 737 (2019).

30. Zhou, $X$. et al. Ribosomal proteins $L 11$ and $L 5$ activate TAp73 by overcoming MDM2 inhibition. Cell Death Differ. 22, 755-766 (2015).

31. Zhang, Y., Zeng, S. X., Hao, Q. \& Lu, H. Monitoring p53 by MDM 2 and MDMX is required for endocrine pancreas development and function in a spatiotemporal manner. Dev. Biol. 423, 34-45 (2017).

32. Liao, J. M. \& Lu, H. ChIP for identification of p53 responsive DNA promoters. Methods Mol. Biol. 962, 201-210 (2013).

33. Bouvard, $\mathrm{V}$. et al. Tissue and cell-specific expression of the p53-target genes: bax, fas, mdm2 and waf1/p21, before and following ionising irradiation in mice. Oncogene 19, 649-660 (2000).

34. Levine, A. J. \& Oren, $M$. The first 30 years of p53: growing ever more complex. Nat. Rev. Cancer 9, 749-758 (2009).

35. Eriksson, S. E., Ceder, S., Bykov, V. J. N. \& Wiman, K. G. p53 as a hub in cellular redox regulation and therapeutic target in cancer. J. Mol. Cell Biol. 11, 330-341 (2019).

36. Hoh, J. et al. The p53MH algorithm and its application in detecting p53responsive genes. Proc. Natl Acad. Sci. USA 99, 8467-8472 (2002).

37. Nguyen, D. T. T. et al. The ubiquitin ligase LIN41/TRIM71 targets p53 to antagonize cell death and differentiation pathways during stem cell differentiation. Cell Death Differ. 24, 1063-1078 (2017).

38. Kemeny, S. et al. Parkin promotes degradation of the mitochondrial proapoptotic ARTS protein. PLOS ONE 7, e38837 (2012).

39. Bornstein, B. et al. X-linked inhibitor of apoptosis protein promotes the degradation of its antagonist, the pro-apoptotic ARTS protein. Int. J. Biochem. Cell Biol. 44, 489-495 (2012).

40. Freed-Pastor, W. A. \& Prives, C. Mutant p53: one name, many proteins. Genes Dev. 26, 1268-1286 (2012).

41. Vassilev, L. T. et al. In vivo activation of the p53 pathway by small-molecule antagonists of MDM2. Science 303, 844-848 (2004). 